\title{
Classificação do estado de conservação de fachadas de edificações públicas
}

\section{Classification of the conservation state of public building facades}

\author{
${ }^{1}$ Ana Maria de Sousa Santana de Oliveira, ${ }^{2}$ Alessandra Bordin Terribele, ${ }^{3}$ Bruna Swiderski, \\ ${ }^{4}$ Ricardo Rocha Oliveira
}

\begin{abstract}
${ }^{1}$ Doutora em Engenharia Civil, professora adjunta da Universidade Estadual do Oeste do Paraná - Universidade Federal de Santa Catarina, R. Universitária, 2069, Cascavel-PR. ${ }^{2}$ Graduanda em Engenharia Civil - Universidade Federal de Santa Catarina, R. Universitária, 2069, Cascavel-PR. ${ }^{3}$ Graduanda em Engenharia Civil - Universidade Federal de Santa Catarina, R. Universitária, 2069, Cascavel-PR. ${ }^{4}$ Doutor em Engenharia Civil, professor adjunto da Universidade Estadual do Oeste do Paraná - Universidade Federal de Santa Catarina, R. Universitária, 2069, Cascavel-PR.
\end{abstract}

\begin{abstract}
RESUMO: As edificações estão sujeitas a diversos fatores, tais como intempéries, uso e desgaste, envelhecimento natural, que podem levar à redução do desempenho e vida útil devido ao surgimento de patologias. Em obras públicas, como as universidades, há o agravante em virtude de serem utilizadas por um grande número de pessoas, com diversas finalidades, aumentando as chances de ocorrências de anomalias. A fim de identificar essas anomalias e orientar a necessidade e urgência de intervenções, existem métodos de avaliação do estado de conservação de edificações. O objetivo deste trabalho foi realizar a classificação do estado de conservação das fachadas da Unioeste Campus Cascavel, a partir da amostra de três blocos: Biblioteca, Centro de Ciências Biológicas e da Saúde - CCBS e Laboratório de Estruturas e Materiais da Engenharia - LEME. Para isto, foi escolhida uma metodologia portuguesa, desenvolvida pelo Laboratório Nacional de Engenharia Civil (LNEC), em Lisboa, denominada Método de Avaliação do Estado de Conservação (MAEC), adaptada para estudos de fachadas. Os resultados indicaram que $67 \%$ das fachadas encontram-se em Bom estado de conservação, $17 \%$ delas classificaram-se como Excelente, $8 \%$ como Médio, 8\% como Ruim e nenhuma delas enquadrou-se como Péssimo. Em relação aos blocos, a Biblioteca resultou em estado de conservação Médio, com índice de anomalias de 3,28, e o CCBS e o LEME classificaram-se como Bom estado de conservação, com índices de anomalias de 3,79 e 4,46, respectivamente. No presente estudo foi possível verificar a abrangência e facilidade no que diz respeito à aplicação do método utilizado para classificação de fachadas, podendo, este, ser utilizado em qualquer tipo de edificação, com vista a se ter uma ferramenta para auxiliar nos processos de manutenção e conservação de fachadas.
\end{abstract}

Palavras Chave: Conservação. Anomalia. Edificações públicas. Sistema de Vedação Vertical Externo.

\begin{abstract}
The buildings are exposed to many factors, such as inclement weather, wear and tear, natural aging, which can lead to performance and lifespan reduction due to the appearance of pathologies. In public buildings, such as universities, there is the aggravation of being used by a large number of people, for different goals, increasing the chances of anomalies' occurrences. With the aim of identifying such anomalies and to guide the urgency and need for interventions, there are evaluation methods of the building's conservation state. The objective of this work was to classify the conservation state of Unioeste Campus Cascavel facades, with a sample of three blocks: Library, Biological and Health Sciences Center - CCBS and Laboratory of Structures and Materials of Engineering - LEME. For this purpose, a Portuguese methodology was chosen, developed by the National Laboratory of Civil Engineering (LNEC), in Lisbon, known as Evaluation Method of Conservation State (MAEC), adapted for facade studies. The results indicated that $67 \%$ of facades are in Good condition, $17 \%$ of them are classified as Excellent, $8 \%$ as Medium, $8 \%$ as Bad, and none of them was qualified as Awful. Regarding the blocks, the Library resulted in a Medium state of conservation, with anomaly index of 3.28, and CCBS and LEME were classified as a Good conservation status, with anomaly indexes of 3.79 and 4.46 , respectively. In the present study it was possible to notice the great comprehensiveness and ease with regard to the application of the method used to classify facades, which can be used in any buildings of interest.
\end{abstract}

Keywords: Conservation. Anomalies. Public building. External Vertical Sealing System.

\section{INTRODUÇÃO}

A necessidade de se abrigar das intempéries do meio ambiente e de predadores é uma preocupação do homem desde os primórdios da humanidade. Após o surgimento das primeiras construções, e com o desenvolvimento de novos materiais e tecnologias, o homem tem buscado, ao longo dos anos, edificações cada vez mais duráveis, sólidas, 
confortáveis e seguras (GUIMARÃES, 2003).

Algum tempo após o término da construção, as edificações entram em processo de degradação, seja por falhas de projeto, de execução, de materiais indevidamente utilizados, uso e operação (OLIVEIRA, 2013).

Sobre isso, Delgado (2013) afirma que o efeito de fatores ambientais, uso e desgaste, somados ao envelhecimento natural, com o passar do tempo levam ao envelhecimento da estrutura, materiais, acabamentos e equipamentos dos edifícios, causando perda de desempenho, trazendo como consequência o surgimento de anomalias nos elementos construtivos do edifício. Entretanto, mesmo com a grande exigência do mercado atual, notase, com frequência, o aparecimento precoce de manifestações patológicas em edifícios recentes. Isso leva à necessidade de intervenções físicas, como a manutenção e a reabilitação. Para a correta identificação das anomalias e indicação das intervenções necessárias, é de fundamental importância a avaliação do estado de conservação de edifícios (OLIVEIRA, 2013b).

Essa avaliação é entendida como uma determinação do nível de conservação, por meio da identificação dos tipos de anomalias presentes e o grau com que se manifestam, obtendo como resultado, através de metodologias específicas, o estado de conservação.

Nesse sentido a conservação pode ser entendida como sinônimo de preservação e oposto de degradação. Uma edificação conservada apresenta desempenho considerado satisfatório atendendo às exigências dos usuários e das normas técnicas, quanto a durabilidade, segurança e conforto (ABNT, 2013).

Os métodos de avaliação do estado de conservação dos edifícios são a base para a implementação de qualquer programa de gestão de um parque edificado, para a definição do estado de conservação de cada edifício e planejamento das intervenções de manutenção, reparação, reabilitação e outras (OLIVEIRA, 2013a).

Para Pedro, Vilhena e Paiva (2012), a avaliação do estado de conservação de um edifício é tarefa tecnicamente complexa, exige conhecimento, equipamento e tempo. Normalmente, o diagnóstico das causas das anomalias não é investigado em profundidade, por meio da identificação de sinais ou indicadores de sintomas visíveis de degradação ou de anomalia.

As metodologias de avaliação de estado de conservação geralmente identificam anomalias existentes nos elementos funcionais, e fazem uma análise comparando as características atuais com as características que o edifício apresentava ao fim de sua construção. Os resultados obtidos nesta análise permitem identificar a urgência da intervenção bem como a técnica mais adequada para a reparação (ALMEIDA, 2012).

Existem diversos métodos de avaliação do estado de conservação de edificações, que analisam a existência e a intensidade das anomalias encontradas. As diferenças entre os métodos estão relacionadas principalmente aos campos de aplicação, objetivos e maneiras de obter o resultado final.

Uma dessas metodologias, denominada de Método de Avaliação do Estado de Conservação (MAEC), foi adaptada por Almeida (2012) para estudos em fachadas. Os elementos que compõem as fachadas dos edifícios são a primeira barreira de proteção contra solicitações, sendo, por essa razão, essencial que sejam mantidos em adequado estado de conservação e desempenho.

Segundo Silva (2014), de todos os elementos constituintes de um edifício, as fachadas ou vedações externas são elementos críticos, já que compõem a envoltória de proteção dos edifícios, atuando como a primeira barreira contra as solicitações causadas por esforços internos e externos, como ação das intempéries. Desse modo, é fundamental que os revestimentos apresentem desempenho dentro dos limites aceitáveis.

Sujeitas à deterioração e degradação, as fachadas das edificações precisam adotar um 
plano de manutenção constante, seja ela preventiva ou corretiva. A fim de atender as necessidades e requisitos de segurança dos usuários, a ABNT (Associação Brasileira de Normas Técnicas) criou uma série de normas que recomendam os passos e critérios que devem ser adotados na gestão da manutenção predial, manutenabilidade e vida útil das edificações. Entre elas, pode-se citar a NBR 15575-1 (ABNT, 2013) que estabelece que todo os componentes, elementos e sistemas devam manter a capacidade funcional durante a vida útil de projeto, sendo necessário para tanto que sejam procedidas intervenções periódicas de manutenção especificadas pelos respectivos fornecedores.

É inviável sob o ponto de vista econômico e inaceitável sob o ponto de vista ambiental considerar as edificações como produtos descartáveis, passíveis da simples substituição por novas construções quando os requisitos de desempenho atingem níveis inferiores àqueles exigidos pela norma NBR 15575-1. Diante do exposto, considera-se que a manutenção das edificações, e em especial suas fachadas sejam realizadas, tão logo colocadas em uso.

A elaboração e a implantação de programas de manutenção nas edificações, assim como a identificação do estado de conservação, além de serem importantes para a segurança e qualidade de vida dos usuários, são essenciais para a manutenção dos níveis de desempenho ao longo da vida útil, apresentados e exigidos pela Norma 15575-1.

Conforme Gaspar e Brito (2005), o adequado diagnóstico do estado de conservação dos elementos de fachada e o seu prognóstico de comportamento servem para auxiliar projetistas e usuários a conceber edificações e estratégias de manutenção que permitam aumentar a durabilidade desses componentes.

Muitas vezes verifica-se grande ocorrência de anomalias em obras públicas, inclusive em edificações universitárias. Devido à grande importância das instituições de ensino superior na sociedade, verifica-se a relevância desse estudo, que teve como objetivo realizar a classificação do estado de conservação das fachadas dos blocos pertecentes à Universidade Estadual do Oeste do Paraná.

\section{MATERIAIS E MÉTODOS}

\subsection{Local de estudo}

O trabalho foi desenvolvido na Universidade Estadual do Oeste do Paraná - Unioeste, no campus de Cascavel - PR, como uma complementação ao trabalho desenvolvido por Menin (2016). O trabalho citado realizou o levantamento e a quantificação da incidência das manifestações patológicas presentes nas edificações da universidade, nas áreas internas e externas dos blocos escolhidos. Em continuidade a este trabalho, foi realizada a classificação do estado de conservação das fachadas das edificações da mesma Universidade, mantendo parcialmente a amostra estudada pelo referido autor.

A Unioeste é uma Instituição de Ensino Superior - (IES), criada em 1994, composta por oito campi, dividida em cinco cidades, Cascavel, Toledo, Foz do Iguaçu, Marechal Cândido Rondon e Francisco Beltrão. O campus de Cascavel é um dos três campi da universidade em Cascavel, que ainda conta com a Reitoria e o Hospital Universitário como parte das instalações na cidade. O campus de Cascavel, objeto deste estudo, está instalado em um terreno de $123.958,17 \mathrm{~m}^{2}$, localizado na região sul da cidade. Deste total, aproximadamente $59 \mathrm{mil} \mathrm{m}^{2}$ correspondem a área construída.

\subsection{Escolha das amostras}

O primeiro critério utilizado foi uma vistoria inicial, em que se identificaram os blocos com quantidades consideráveis de manifestações patológicas, visando adequadamente identificar a avaliação do estado de conservação blocos. 
O segundo foi a realização de um teste piloto, em que foi escolhido um bloco aleatório e aplicado o instrumento de pesquisa desenvolvido em algumas das fachadas. Esse teste objetivou verificar a aplicabilidade do instrumento de pesquisa, assim como analisar a facilidade no uso e interpretação do instrumento. Durante este teste, identificou-se os prédios que melhor se comportariam a metodologia empregada.

Como critério exclusão, considerou-se a ocorrência de manutenções recentes nas fachadas, como é o caso dos blocos da antiga FECIVEL - Faculdade de cascavel e da Reitoria, sendo que, por esta razão, foram desconsiderados da amostragem.

Dessa forma, a amostra escolhida foi composta por três prédios do campus de Cascavel: Biblioteca (1), Centro de Ciências Biológicas e da Saúde - CCBS (2) e Laboratório de Estruturas e Materiais da Engenharia - LEME (3), conforme ilustrado na Figura 1.

Figura 1 - Edificações utilizadas como objeto de estudo e localização dos blocos da amostra

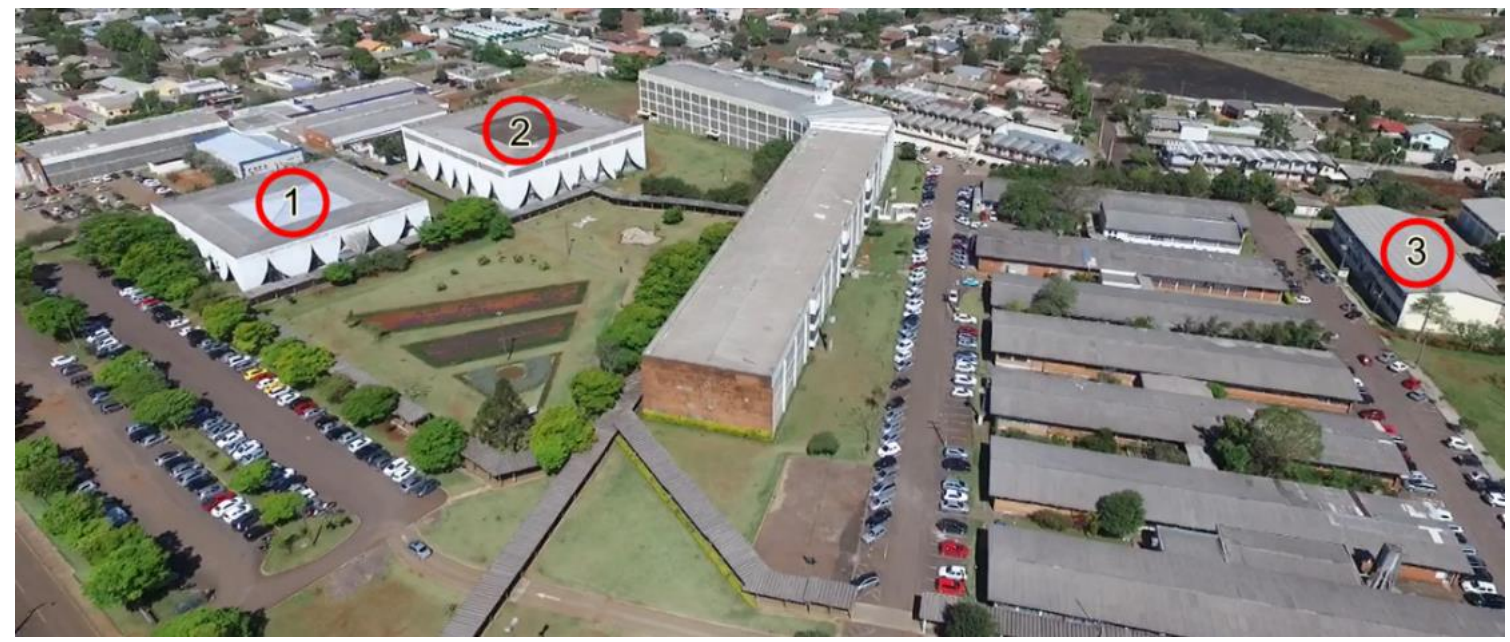

\subsection{Método de Avaliação do Estado de Conservação - MAEC}

O MAEC, desenvolvido pelo LNEC em 2006, em Lisboa, Portugal, visa determinar com rigor, objetividade e transparência o estado de conservação de imóveis (podendo avaliar um edifício parcialmente ou em sua totalidade) e a existência de infraestruturas básicas (VILHENA, 2007).

O rigor deve-se ao fato de que o imóvel passa por uma avaliação minuciosa de suas condições. A objetividade decorre das regras claras e pré-definidas, permitindo que os resultados sejam tão independentes quanto possível do técnico que realiza a avaliação. E a transparência assegura que o processo e os resultados sejam facilmente entendidos por todos os envolvidos.

O estado de conservação avalia os níveis de anomalias que afetam os elementos funcionais do imóvel, comparando as condições atuais, no momento da inspeção, com as condições que o imóvel apresentava quando foi construído ou quando passou pela última intervenção.

Em sua forma original, o instrumento de pesquisa utilizado pelo método é uma Ficha de Avaliação que divide a edificação em 37 elementos funcionais e dependendo do tipo de edifício, alguns elementos podem ser considerados como não aplicáveis. No entanto, para esta pesquisa foi utilizada uma adaptação do método para o estudo de fachadas criado por Almeida (2012), onde se descartou a avaliação dos ambientes internos.

O método de análise e cálculo manteve-se igual ao método original, porém, foi necessário realizar alterações na Ficha de Avaliação, em que foram retirados alguns itens, 
restringindo aos elementos que compõem a fachada e elementos que sejam incorporados a ela, como caixilhos e juntas de dilatação.

A Ficha de Avaliação foi reduzida a sete elementos funcionais, conforme Quadro 1.

Quadro 1 - Elementos funcionais adaptados e suas respectivas ponderações

\begin{tabular}{clc}
\hline Item & Elemento Funcional & Ponderação \\
\hline 1 & Elementos estruturais & 6 \\
\hline 2 & Alvenaria & 4 \\
\hline 3 & Revestimento & 4 \\
\hline 4 & Acabamento & 5 \\
\hline 5 & Juntas & 3 \\
\hline 6 & Esquadrias & 3 \\
\hline 7 & Elementos salientes & \\
\hline
\end{tabular}

Fonte: Almeida (2012)

O método realiza a determinação das condições das fachadas por meio da ponderação de cada elemento presente na Ficha de Avaliação, de acordo com sua importância para o funcionamento do edifício, variando de 1 a 6 . Elementos muito importantes recebem ponderação 5 ou 6, os importantes, 3 ou 4, e os considerados pouco importantes, 1 ou 2. Notase que apenas o elemento estrutura recebe ponderação 6, devido a sua grande importância no edifício (Quadro 1).

A função do avaliador é determinar, para cada elemento, um nível de anomalia, de "Muito Simples" até "Muito Grave". Para cada nível, há um nível de conservação associado, variando de 1 (Muito Grave) a 5 (Muito Simples).

A classificação das anomalias nos níveis anteriormente citados é orientada pelo uso do Quadro 2, segundo os critérios de consequência da anomalia na satisfação das exigências funcionais e tipo e extensão do trabalho necessário para a correção da anomalia.

Quadro 2 - Classificação do nível de anomalia

\begin{tabular}{|c|c|}
\hline Muito simples & Ausência de anomalias ou anomalias sem significado. \\
\hline Simples & $\begin{array}{l}\text { Anomalias que prejudicam o aspecto estético, e que requerem trabalhos de fácil } \\
\text { execução. }\end{array}$ \\
\hline \multirow{2}{*}{ Médias } & $\begin{array}{l}\text { Anomalias que prejudicam o aspecto estético, e que requerem trabalhos de difícil } \\
\text { execução. }\end{array}$ \\
\hline & $\begin{array}{l}\text { Anomalias que prejudicam o uso e conforto e requerem trabalhos de limpeza, } \\
\text { substituição ou reparação de fácil execução. }\end{array}$ \\
\hline \multirow{2}{*}{ Graves } & $\begin{array}{l}\text { Anomalias que prejudicam o uso e conforto e requerem trabalhos de limpeza, } \\
\text { substituição ou reparação de difícil execução. }\end{array}$ \\
\hline & $\begin{array}{l}\text { Anomalias que colocam em risco a saúde e/ou a segurança, podendo motivar } \\
\text { acidentes sem gravidade, e que requerem trabalhos de fácil execução. }\end{array}$ \\
\hline \multirow{3}{*}{ Muito graves } & $\begin{array}{l}\text { Anomalias que colocam em risco a saúde e/ou a segurança, podendo motivar } \\
\text { acidentes sem gravidade, e que requerem trabalhos de difícil execução. }\end{array}$ \\
\hline & $\begin{array}{l}\text { Anomalias que colocam em risco a saúde e/ou segurança, podendo motivar acidentes } \\
\text { graves ou muito graves. }\end{array}$ \\
\hline & Ausência ou inoperacionalidade de infraestrutura básica. \\
\hline
\end{tabular}
Fonte: Adaptado de Almeida (2012).

A indicação do estado de conservação de cada local em que é aplicado o instrumento é atribuída de acordo com o cálculo do índice de anomalias. Para cada elemento, realiza-se a multiplicação da ponderação pelo nível de conservação correspondente ao nível de anomalia 
atribuído pelo avaliador. A soma desses produtos é então dividida pela soma das ponderações dos elementos considerados como aplicáveis, conforme Equação 1.

$I A=\frac{\sum(\text { Ponderação } x \text { Nível de Conservação })}{\sum \text { Ponderações }}$

O cálculo resulta no índice de anomalias (IA), que varia entre 1 e 5 . O estado de conservação correspondente, de acordo com o índice de anomalias, é indicado pelo Quadro 3.

Quadro 3 - Correspondência entre índice de anomalias e estado de conservação

\begin{tabular}{|c|c|c|c|c|c|}
\hline Índice de anomalias & $\begin{array}{c}5,00 \geq \mathrm{IA} \geq \\
4,50\end{array}$ & $\begin{array}{c}4,50>\mathrm{IA} \geq \\
3,50\end{array}$ & $\begin{array}{c}3,50>\mathrm{IA} \geq \\
2,50\end{array}$ & $\begin{array}{c}2,50>\mathrm{IA} \geq \\
1,50\end{array}$ & $\begin{array}{c}1,50>\mathrm{IA} \geq \\
1,00\end{array}$ \\
\hline Estado de conservação & Excelente & Bom & Médio & Ruim & Péssimo \\
\hline
\end{tabular}

Fonte: Adaptado de MAEC (2006) e Vilhena (2007)

Há ainda duas regras quanto ao valor final do índice de anomalias, que evitam que haja um falso julgamento no caso de haver um alto desvio padrão em relação à média. Isso pode ocorrer quando há alguns elementos com estados de conservação muito bons e outros muito ruins, já que o resultado seria mediano, mascarando o real estado de conservação. Assim, deve-se verificar as seguintes condições:

I - Se há algum elemento funcional de ponderação a partir de 3 com estado de conservação inferior em mais de uma unidade ao estado de conservação do imóvel; caso isso ocorra, este deve ser reduzido ao nível imediatamente superior ao estado de conservação do elemento funcional de ponderação a partir de 3 em pior estado:

$$
\mathrm{Se} \mathrm{EC}_{\text {elemento }}<\mathrm{EC}_{\text {imóvel }}-1 \rightarrow \mathrm{EC}_{\text {imóvel }}=\mathrm{EC}_{\text {elemento }}+1 \text {; }
$$

II - Se há algum elemento funcional de ponderação até 2 com estado de conservação inferior em mais de duas unidades ao estado de conservação do imóvel; caso isso ocorra, este deve ser reduzido ao nível superior em duas unidades ao estado de conservação do elemento funcional de ponderação até 2 em pior estado:

$$
\text { Se } \mathrm{EC}_{\text {elemento }}<\mathrm{EC}_{\text {imóvel }}-2 \rightarrow \mathrm{EC}_{\text {imóvel }}=\mathrm{EC}_{\text {elemento }}+2 \text {. }
$$

Após classificados o estado de conservação de cada fachada, calculou-se o Índice de Anomalias do Bloco (IABloco), por meio de média simples (Equação 4). No caso da existência de fachadas com subdivisões, este índice foi calculado a partir da média simples das subdivisões (Equação 5), para então ser usado no cálculo do índice de anomalias do bloco.

$$
\begin{aligned}
& \mathrm{IA}_{\mathrm{BLOCO}}=\left(\mathrm{IA}_{\mathrm{A}}+\mathrm{IA}_{\mathrm{B}}+\mathrm{IA}_{\mathrm{C}}+\mathrm{IA}_{\mathrm{D}}\right) / 4 \\
& \mathrm{IA}_{\mathrm{SUB}}=\left(\mathrm{IA}_{\mathrm{D} 1}+\mathrm{IA} \mathrm{A}_{\mathrm{D} 2}+\mathrm{IA}_{\mathrm{D} 3}+\ldots .+\mathrm{I} \mathrm{A}_{\mathrm{Dn}}\right) / \mathrm{n}
\end{aligned}
$$




\subsection{Coleta de dados}

A coleta dos dados foi realizada durante o ano de 2017, por meio de vistorias nos blocos e, simultaneamente, foi feita a aplicação e o preenchimento das fichas de avaliação.

A ficha de avaliação consiste num primeiro momento na descrição das características da amostra, tais como: endereço, nome do bloco, código do bloco, código da fachada, orientação geográfica, etc. Posteriormente, é realizada a classificação dos elementos funcionais e em seguida, de acordo com as ponderações citadas no Quadro 1, calculado o índice de anomalias. Por fim, é feita a avaliação, classificando o estado de conservação.

Foram analisadas $100 \%$ das fachadas dos blocos escolhidos para a amostra, já que não houve restrição de acesso a esses locais, por serem apenas ambientes externos.

Para facilitar a análise, as fachadas dos blocos foram divididas de acordo com suas orientações geográficas, recebendo códigos alfabéticos, de A a D. A cada fachada geográfica foi atribuída uma ficha, a qual foi analisada individualmente. No caso da Biblioteca e do CCBS, uma das fachadas sofreu subdivisões, devido a sua descontinuidade. Essas divisões são ilustradas na Figura 2.

Figura 2 - Códigos das fachadas dos blocos

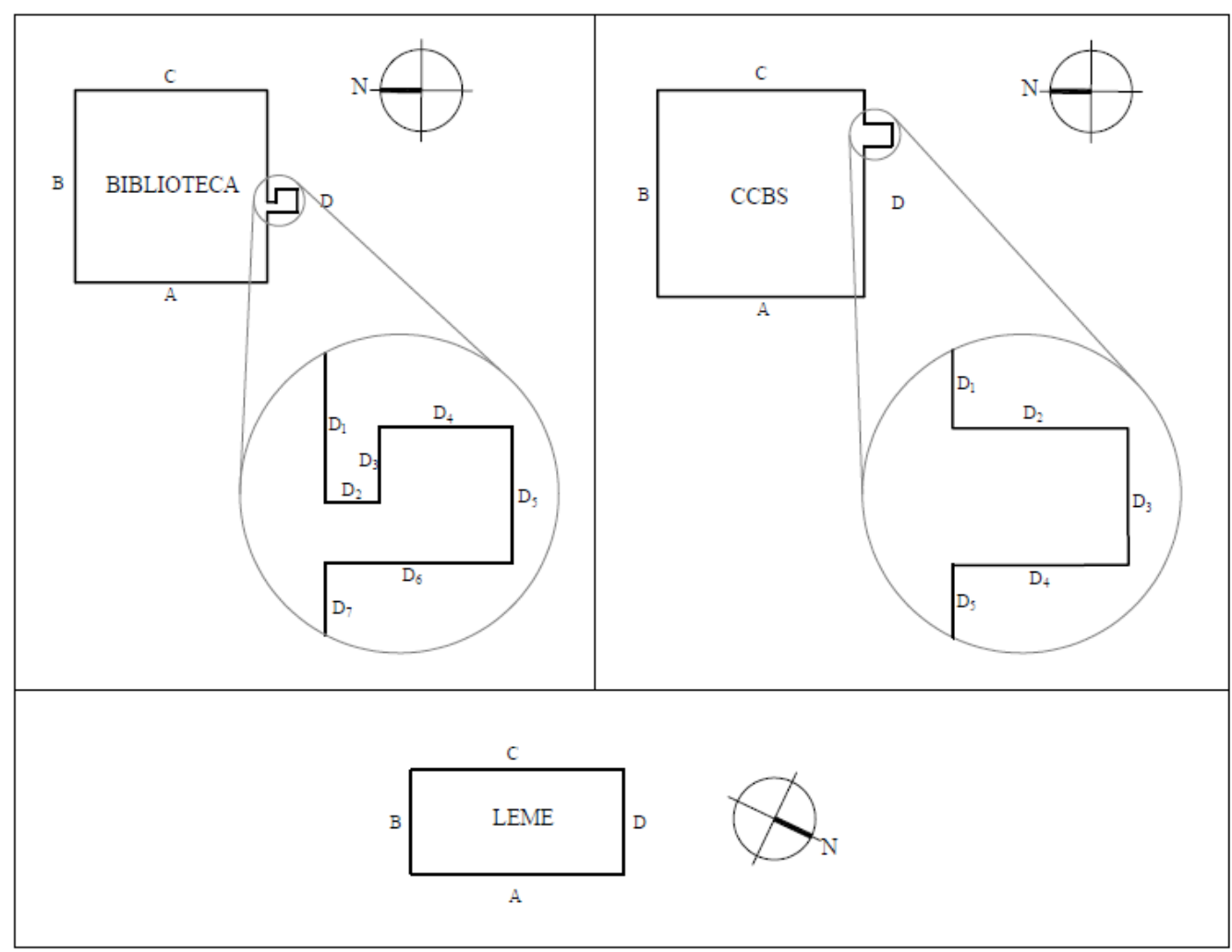

Nas vistorias realizadas no bloco Biblioteca, houve certa dificuldade na análise dos elementos arquitetônicos conhecidos como pétalas, conforme croqui apresentado na Figura 3. Nesse caso, decidiu-se que as pétalas seriam analisadas como elementos salientes, já que apresentam detalhes arquitetônicos ressaltados em relação à fachada do bloco.

Essa consideração também foi utilizada para o bloco do CCBS, que também possui as pétalas como componentes de sua fachada. 
Figura 3 - Representação da divisão das pétalas em diferentes elementos salientes

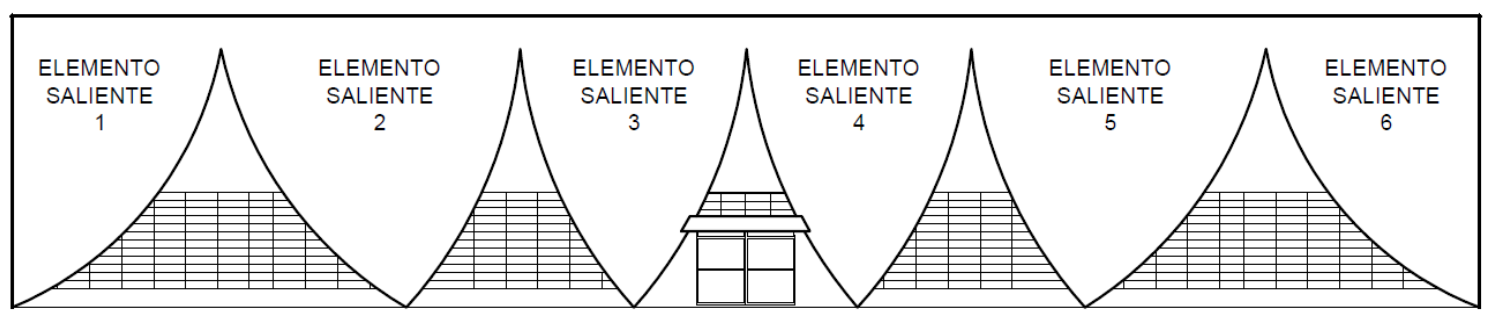

Para a coleta de dados, houve o cuidado de realizar as análises sempre com condições climáticas semelhantes, portanto todas as vistorias foram realizadas em dias não chuvosos e sem chuva há pelo menos dois dias, e com temperaturas entre $25^{\circ} \mathrm{C}$ e $30^{\circ} \mathrm{C}$.

Foi feito registro fotográfico das anomalias encontradas, tanto para comprovação da classificação atribuída, como para sanar possíveis dúvidas que pudessem surgir ao longo do trabalho, muitas vezes evitando a necessidade de retornar ao bloco e realizar nova vistoria.

Por se tratar de um processo que depende da avaliação do pesquisador, este acaba sendo parcialmente subjetivo, apesar de existirem parâmetros bem definidos que procuram deixar o método o mais objetivo possível. Por esse motivo, depois de realizadas todas as vistorias, foi feita uma análise se os critérios utilizados foram os mesmos para todos os blocos, retornando brevemente aos blocos, para conferir os dados coletados.

\section{RESULTADOS}

Os resultados serão apresentados divididos por blocos, sendo apontadas as anomalias encontradas em cada um deles, porém sem especificar a qual fachada se refere. Após cada anomalia, entre parênteses, são indicados os níveis de anomalia atribuídos, conforme os critérios do Quadro 2. Na maioria dos blocos, as anomalias se repetem em todas as fachadas.

Vale ressaltar que a metodologia utilizada avalia o nível de anomalias em função de sua gravidade, sendo, portanto, um método qualitativo. Dessa forma, não importa a quantidade e extensão das manifestações patológicas, mas sim a qualidade das mesmas, ou seja, os aspectos afetados: estético, uso e conforto ou saúde e segurança.

Por esse motivo, em muitas das fachadas, a deterioração é esteticamente acentuada, porém, do ponto de vista do método, os resultados acabam apresentando valores de gravidade baixa, com estado de conservação Bom em muitos casos.

A resolução definitiva de algumas dessas manifestações patológicas revela-se, por vezes, difícil de se realizar, já em outras com o custo muito elevado para reparação. Nesse sentido, a adoção, no processo construtivo, de medidas construtivas que minimizem a ocorrência de anomalias será sempre uma boa opção, uma vez que de um lado traz o aumento de qualidade e por outro a diminuição de custos com a manutenção.

Considera-se que para uma melhor execução, é recomendável a utilização das orientações da norma NBR 15.575 (ABNT, 2013), que trata do desempenho das edificações, e tem como objetivo atender as necessidades dos usuários da edificação, dentro de determinadas condições de exposição, ao longo de uma vida útil de projeto e no contexto do ambiente regulatório, econômico e social brasileiro.

Esta norma é uma ferramenta para o usuário estabelecer programas de manutenção corretiva e preventiva. Nesse sentido, um plano de manutenção é fundamental para garantir o desempenho adequado da edificação e dos seus componentes, como as fachadas. 
OLIVEIRA, ANA MARIA; et al; Classificação do estado de conservação de fachadas de edificações públicas. E\&S - Engineering and Science, 2018, 7:3.

\subsection{Biblioteca}

A Biblioteca do campus de Cascavel foi construída em 1997 e ocupa uma área de $4.267 \mathrm{~m}^{2}$, distribuídos em dois pisos. Dispõe de um hall para a realização de eventos de artistas locais, além de ambientes para estudo e um acervo de aproximadamente 36 mil livros. O bloco da biblioteca apresenta elementos de fachada conhecidos como "pétalas", um detalhe arquitetônico que é uma particularidade de alguns blocos da Unioeste (CCBS e Reitoria, além da biblioteca). A Figura 4 representa uma imagem deste bloco.

Figura 4 - Biblioteca - Campus Unioeste

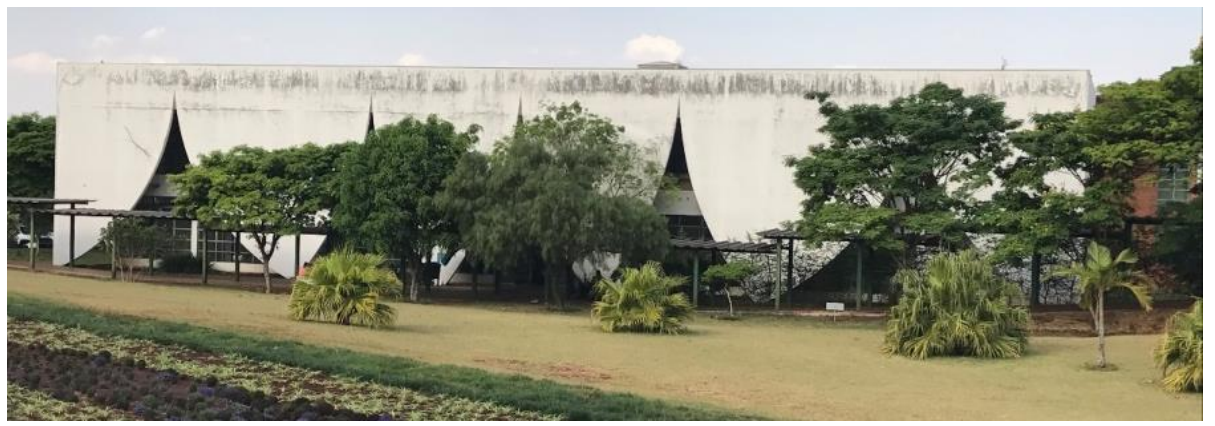

No bloco da Biblioteca foram encontradas as seguintes anomalias: fissuras pontuais no acabamento (Simples), descolamentos e bolor no acabamento (Média), ferrugem nas esquadrias (Simples), fissuras mapeadas no acabamento (Média), descolamento em placas do revestimento cerâmico em altura (Muito Grave) e destacamento entre esquadria e alvenaria (Grave). Estas manifestações estavam presentes em praticamente todas as fachadas deste bloco. Nas Figuras 5 e 6 são ilustradas algumas dessas patologias.

Figura 5 - Incidência de fissuras, bolor e descolamento do revestimento cerâmico
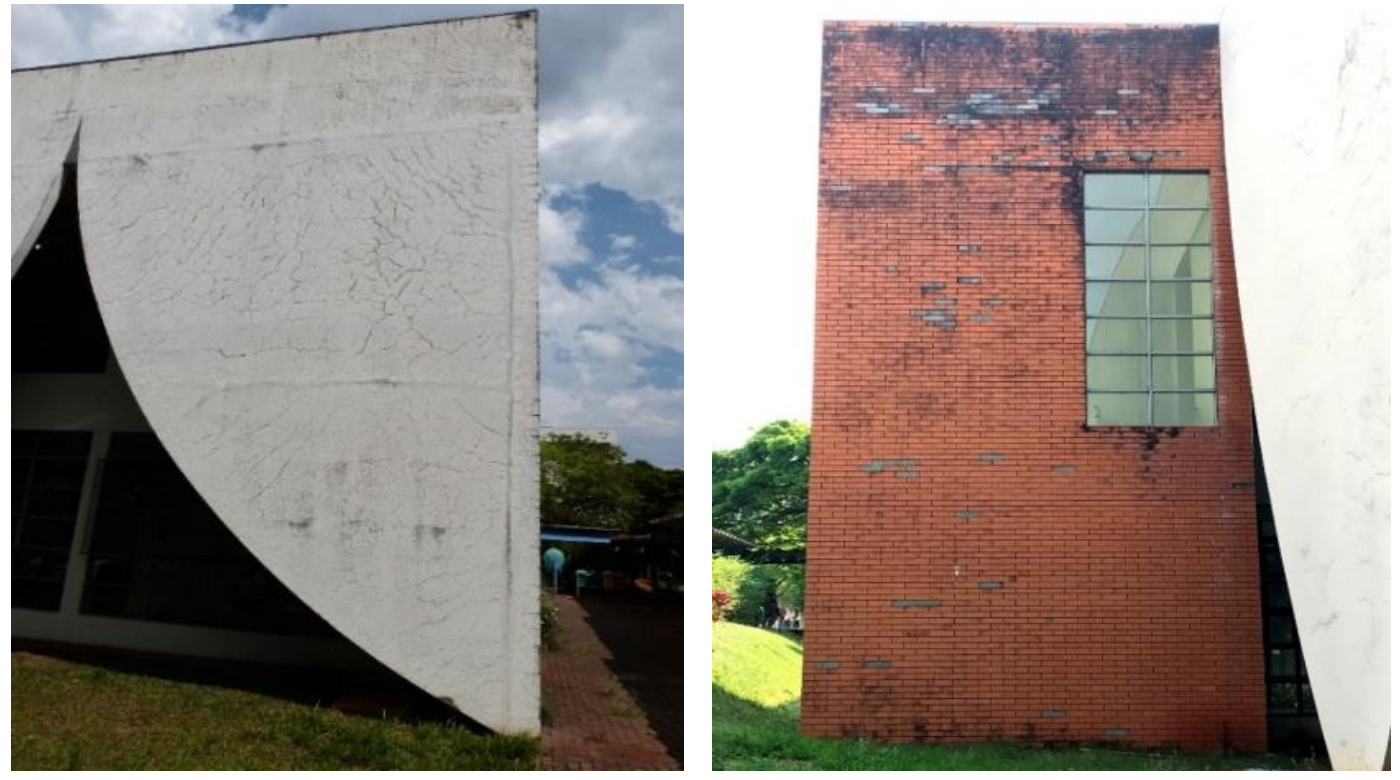
Figura 6 - Descolamento entre esquadria e alvenaria, na fachada da biblioteca

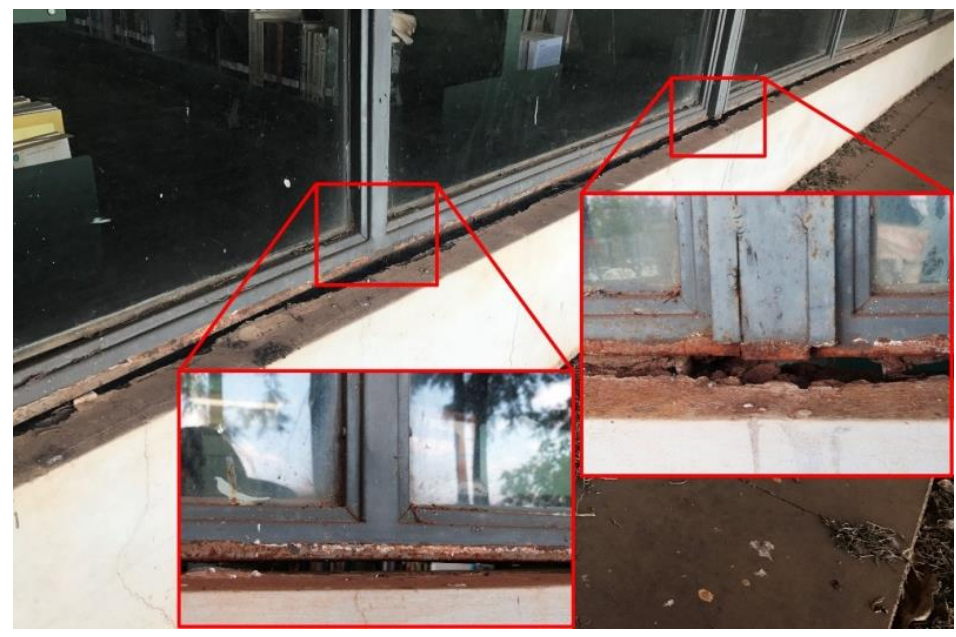

No Quadro 4 são apresentados os índices de anomalia de cada fachada da biblioteca, assim como o índice corrigido, nos casos em que foi necessário realizar tal correção.

Quadro 4 - Índices de anomalia e índice corrigido das fachadas da biblioteca

\begin{tabular}{|c|c|c|c|c|c|c|c|c|c|c|}
\hline \multicolumn{11}{|c|}{ BIBLIOTECA } \\
\hline FACHADA & A & B & $\mathrm{C}$ & D1 & D2 & D3 & D4 & D5 & D6 & D7 \\
\hline SOMA DAS PONTUAÇÕES & 156 & 156 & 144 & 129 & 102 & 86 & 90 & 87 & 87 & 120 \\
\hline SOMA DAS PONDERAÇÕES & 40 & 40 & 40 & 31 & 22 & 19 & 22 & 22 & 22 & 28 \\
\hline ÍNDICE DE ANOMALIAS (IA) & 3,90 & 3,90 & 3,60 & 4,16 & 4,64 & 4,53 & 4,09 & 3,95 & 3,95 & 4,29 \\
\hline MENOR ÍNDICE DE CONSERVAÇÃO & 3 & 3 & 1 & 3 & 4 & 4 & 1 & 1 & 1 & 3 \\
\hline NECESSITA CORREÇÃO? & NÃO & NÃO & SIM & SIM & NÃO & $\mathrm{NÃO}$ & SIM & SIM & SIM & SIM \\
\hline ÍNDICE DE ANOMALIAS CORRIGIDO & - & - & 2,00 & $\mathbf{4 , 0 0}$ & - & - & 2,00 & 2,00 & 2,00 & 4,00 \\
\hline ESTADO DE CONSERVAÇÃO (EC) & $\mathbf{B}$ & $\mathbf{B}$ & $\mathbf{R}$ & B & $\mathbf{E}$ & $\mathbf{E}$ & $\mathbf{R}$ & $\mathbf{R}$ & $\mathbf{R}$ & B \\
\hline
\end{tabular}

Legenda do EC: E - Excelente; B - Bom; M - Médio; R - Ruim; P - Péssimo

Assim, o índice de anomalias da biblioteca resultou em 3,28, caracterizando-a com um estado de conservação Médio.

\subsection{Centro de Ciências Biológicas e da Saúde-CCBS}

O Centro de Ciências Biológicas e da Saúde - CCBS conta com $7.065 \mathrm{~m}^{2}$ e foi construído em 1997. O bloco é formado basicamente de laboratórios para a realização de atividades práticas de formação dos acadêmicos dos cursos da área da saúde (Ciências Biológicas, Enfermagem, Farmácia, Fisioterapia, Medicina e Odontologia) além de salas de aula. É composto também por três órgãos suplementares: herbário, biotério e Instituto de Pesquisa e Pós-Graduação em Odontologia. A Figura 7 apresenta uma imagem do CCBS.

Figura 7 - Imagem do Centro de Ciências Biológicas e da Saúde - CCBS

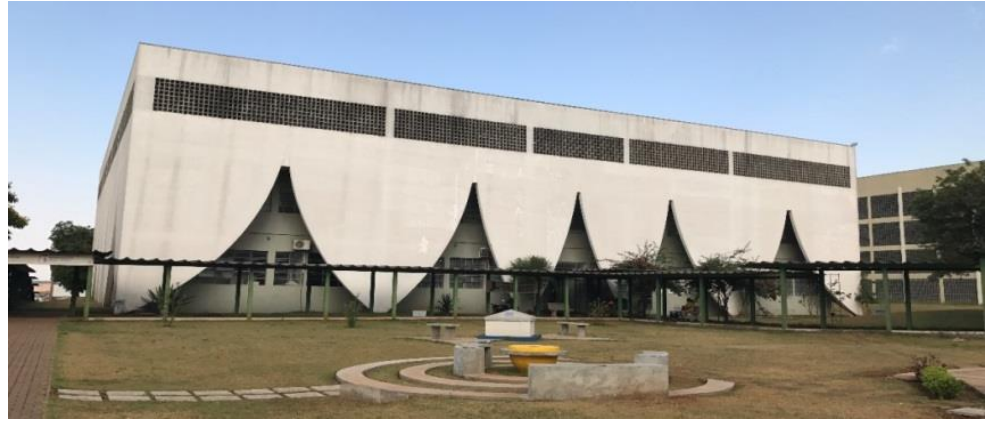


A fachada A do CCBS é a fachada principal, ou seja, de entrada do bloco, voltada para oeste. No bloco foram encontradas anomalias consideradas simples e média. Entre elas: fissuras pontuais no acabamento (Simples), descolamentos e bolor no acabamento (Média), manchas no acabamento (Média), ferrugem nas esquadrias (Simples), vidros quebrados e danificados (Média), fissuras mapeadas no acabamento (Média). Nas Figuras 8 e 9 são ilustradas algumas dessas patologias.

Figura 8 - Incidência de fissuras, manchas no acabamento e vidros quebrados
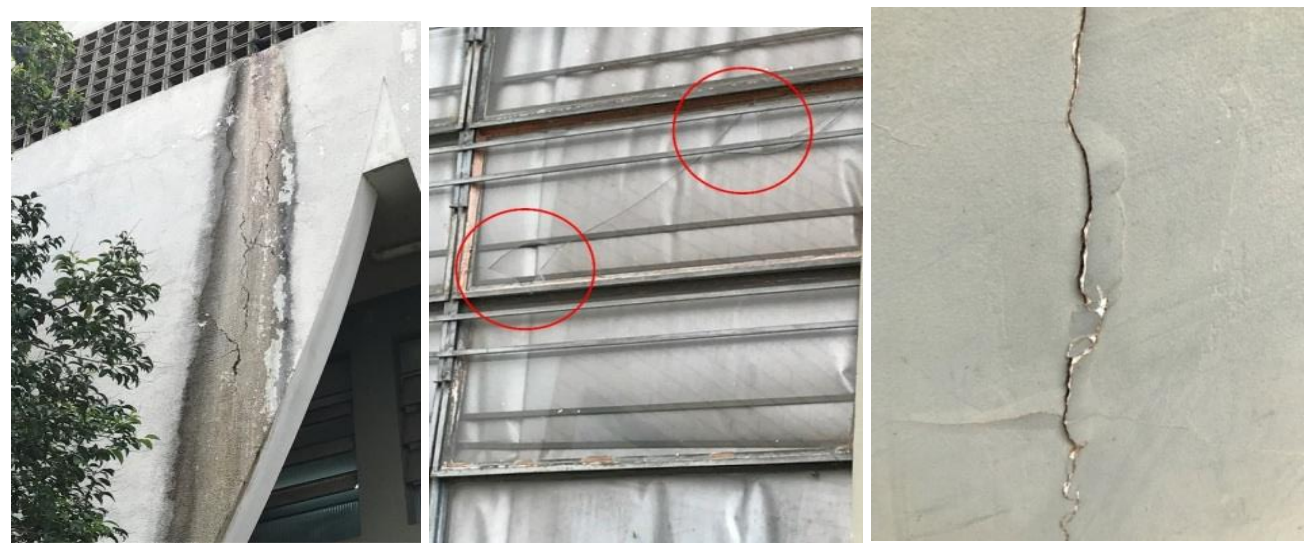

Figura 9 - Incidência de bolor e fissuras e descolamento no acabamento

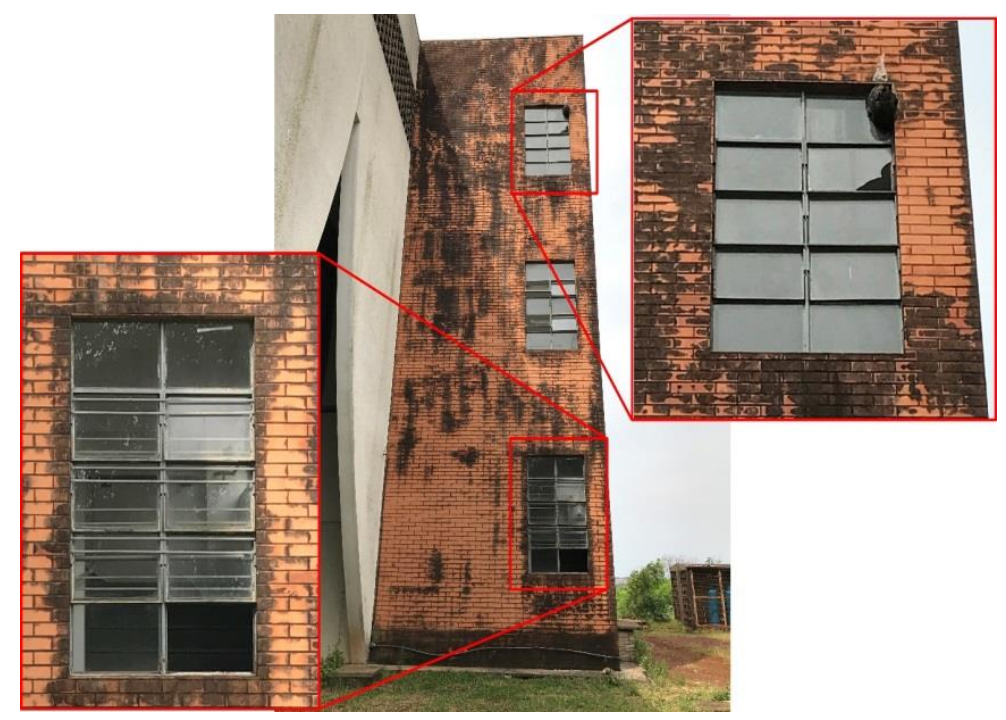

No Quadro 5 são apresentados os índices de anomalia de cada fachada do CCBS, assim como o índice corrigido, nos casos em que foram necessários realizar tal correções.

Quadro 5 - Índices de anomalia das fachadas do CCBS

\begin{tabular}{|c|c|c|c|c|c|c|c|c|}
\hline \multicolumn{1}{|c}{ CCBS } & A & B & C & D1 & D2 & D3 & D4 & D5 \\
\hline FACHADA & 147 & 156 & 144 & 108 & 90 & 94 & 94 & 103 \\
\hline SOMA DAS PONTUAÇÕES & 40 & 40 & 40 & 25 & 22 & 22 & 22 & 25 \\
\hline SOMA DAS PONDERAÇÕES & $\mathbf{3 , 6 8}$ & $\mathbf{3 , 9 0}$ & $\mathbf{3 , 6 0}$ & 4,32 & 4,09 & 4,27 & 4,27 & 4,12 \\
\hline IINDICE DE ANOMALIAS (IA)
\end{tabular}


OLIVEIRA, ANA MARIA; et al; Classificação do estado de conservação de fachadas de edificações públicas. E\&S - Engineering and Science, 2018, 7:3.

Quadro 5 - Índices de anomalia das fachadas do CCBS continuação

\begin{tabular}{|c|c|c|c|c|c|c|c|c|}
\hline \multicolumn{9}{|c|}{ CCBS } \\
\hline FACHADA & $\mathbf{A}$ & B & $\mathbf{C}$ & D1 & D2 & D3 & D4 & D5 \\
\hline MENOR ÍNDICE DE CONSERVAÇÃO & 3 & 3 & 3 & 3 & 3 & 3 & 3 & 3 \\
\hline NECESSITA CORREÇÃO? & $\mathrm{NÃO}$ & $\mathrm{NÃO}$ & NÃO & SIM & SIM & SIM & SIM & SIM \\
\hline ÍNDICE DE ANOMALIAS CORRIGIDO & - & - & - & 4,00 & 4,00 & 4,00 & 4,00 & 4,00 \\
\hline ESTADO DE CONSERVAÇÃO (EC) & B & B & B & B & B & B & B & B \\
\hline
\end{tabular}

Legenda do EC: E - Excelente; B - Bom; M - Médio; R - Ruim; P - Péssimo.

Após classificados o estado de conservação de cada fachada, também foi calculado o índice de anomalias do bloco, por meio de média simples (Equação 4 e 5). Assim, o índice de anomalias do CCBS resultou em 3,79, caracterizando-o com um estado de conservação Bom.

\subsection{Laboratório de Estruturas e Materiais - LEME}

A edificação conta com $1.121 \mathrm{~m}^{2}$ e foi construída para auxiliar no processo de formação dos acadêmicos de Engenharia Civil e Engenharia Agrícola. O bloco apresenta dois pisos, no térreo possui salas de aula e laboratórios para a realização de atividades práticas e projetos de pesquisa e, no segundo piso, abriga salas de professores. Nesse bloco também se encontram as instalações do Programa de Pós-Graduação em Engenharia Agrícola - PPGEC da Universidade. Construído em 2007, possui $17 \mathrm{~m}$ de largura por $45 \mathrm{~m}$ de comprimento e apresenta como particularidade o seu sistema construtivo realizado em pré-moldado com cobertura de fibrocimento.

Nesse bloco foram encontradas as menores incidências de anomalias, se comparado aos demais pesquisados. Entre os blocos observados anteriormente, este aparentou ser o melhor conservado. O mesmo foi incluído na pesquisa pelo fato de possuir fachadas contínuas e bem definidas, com vários elementos, em que se verificou a boa aplicabilidade do instrumento. A Figura 10 representa uma imagem do bloco.

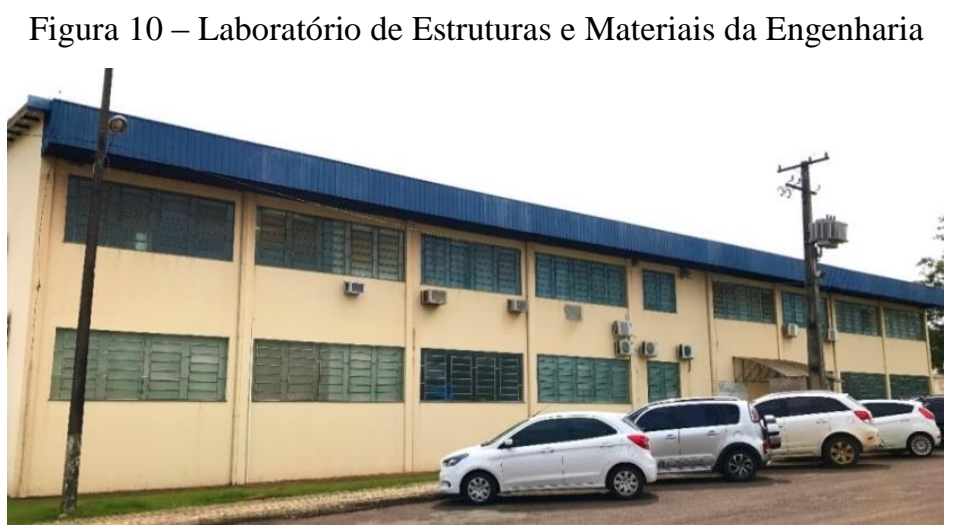

As seguintes anomalias foram identificadas: fissuras pontuais no acabamento (Simples), descolamentos do revestimento (Simples), descolamentos do acabamento (Simples), manchas no acabamento (Simples), ferrugem nas esquadrias (Média), vidros quebrados (Média). Na Figura 11 estão ilustradas algumas dessas patologias. 
OLIVEIRA, ANA MARIA; et al; Classificação do estado de conservação de fachadas de edificações públicas. E\&S - Engineering and Science, 2018, 7:3.

Figura 11 - Incidência de fissuras e descolamentos no acabamento, descolamento do revestimento e vidro quebrado

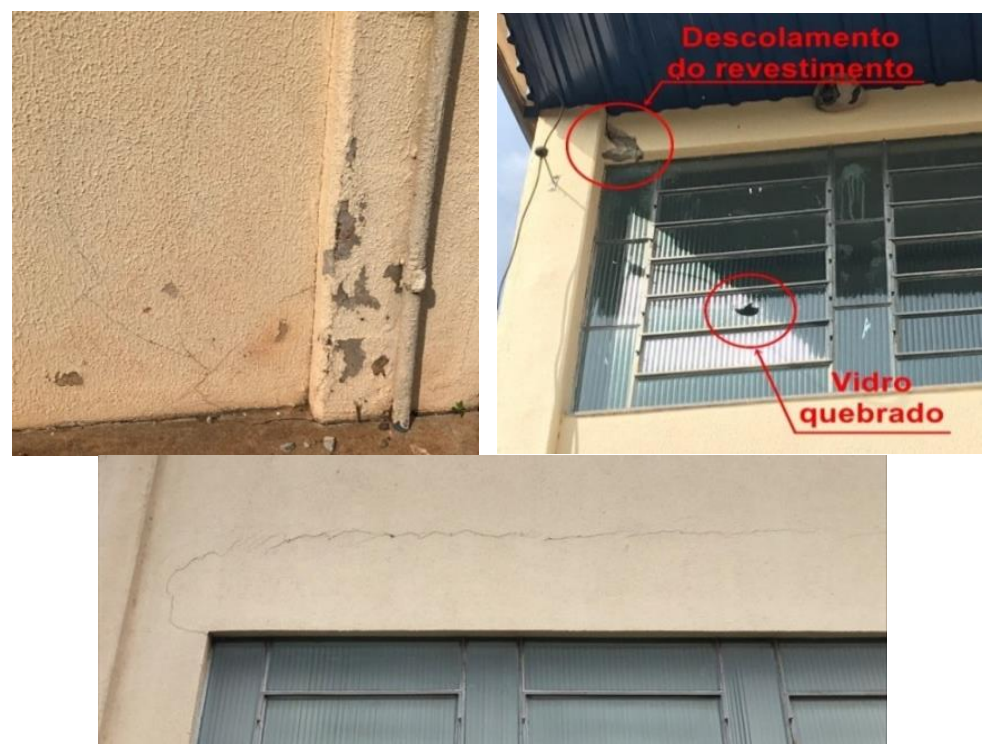

No Quadro 6 são apresentados os índices de anomalia de cada fachada do LEME, assim como o índice corrigido, nos casos em que foi necessário realizar correção.

Quadro 6 - Índices de anomalia das fachadas do LEME

\begin{tabular}{|c|c|c|c|c|}
\hline LEME & A & B & C & D \\
\hline FACHADA & 110 & 117 & 110 & 105 \\
\hline SOMA DAS PONTUAÇÕES & 25 & 25 & 25 & 22 \\
\hline SOMA DAS PONDERAÇÕES & $\mathbf{4 , 4 0}$ & $\mathbf{4 , 6 8}$ & 4,40 & $\mathbf{4 , 7 7}$ \\
\hline ÍNDICE DE ANOMALIAS (IA) & 4 & 4 & 3 & 4 \\
\hline MENOR ÍNDICE DE CONSERVAÇÃO & NÃO & NÃO & SIM & NÃO \\
\hline NECESSITA CORREÇÃO? & - & - & $\mathbf{4 , 0 0}$ & - \\
\hline ÍNDICE DE ANOMALIAS CORRIGIDO & $\mathbf{B}$ & $\mathbf{E}$ & $\mathbf{B}$ & $\mathbf{E}$ \\
\hline ESTADO DE CONSERVAÇÃO (EC)
\end{tabular}

Legenda do EC: E - Excelente; B - Bom; M - Médio; R - Ruim; P - Péssimo

Após classificados o estado de conservação de cada fachada, também foi calculado o índice de anomalias do bloco, por meio de média simples (Equação 4). Assim, o índice de anomalias do LEME resultou em 4,46, caracterizando-o com um estado de conservação Bom.

\subsection{Estado de conservação de todas as edificações da amostra}

Para calcular o índice de anomalias geral de todas as edificações, foi realizada a média simples dos índices de anomalia dos três blocos.

Quadro 7 - Índice de anomalias e estado de conservação dos blocos estudados

\begin{tabular}{cccc}
\hline $\begin{array}{c}\text { Código do } \\
\text { bloco }\end{array}$ & Nome do Bloco & $\begin{array}{c}\text { Índice de } \\
\text { Anomalias }\end{array}$ & $\begin{array}{c}\text { Estado de } \\
\text { Conservação }\end{array}$ \\
\hline 1 & Biblioteca & 3,31 & Médio \\
\hline 2 & CCBS & 3,79 & Bom \\
\hline 3 & LEME & 4,46 & Bom \\
\hline
\end{tabular}


O índice de anomalias geral de todas as edificações resultou em 3,84, caracterizando um estado de conservação Bom para as edificações analisadas.

Ao comparar a quantidade de manifestações patológicas encontradas e as considerações do método utilizado, o estudo indicou que apesar da existência de inúmeras manifestações patológicas nos blocos, dos mais variados níveis, ainda em relação à conservação, os blocos da universidade apresentam-se bem preservados e em bom estado.

Em linhas gerais, o atual estado da degradação encontrado nas fachadas da edificação, é reflexo da ação das condições ambientais aliados ao seu baixo nível de manutenção. Diante disso, seria importante observar o que preconiza a NBR 15575 (ABNT 2013), onde se salienta que um processo de manutenção preventiva, seria normalmente a forma mais econômica e eficaz de minimizar as consequências dessas patologias.

\subsection{Percentual das fachadas por estado de conservação}

Durante a realização deste trabalho, conforme descrito no item 2.4, foram identificados o estado de conservação de cada fachada individualmente. Essa análise permitiu identificar como estão conservadas e o grau de deterioração das mesmas.

A partir dos resultados foram calculados os percentuais das fachadas classificadas em cada estado de conservação, conforme apresentado na Figura 12.

Figura 12 - Percentual de fachadas por estado de conservação

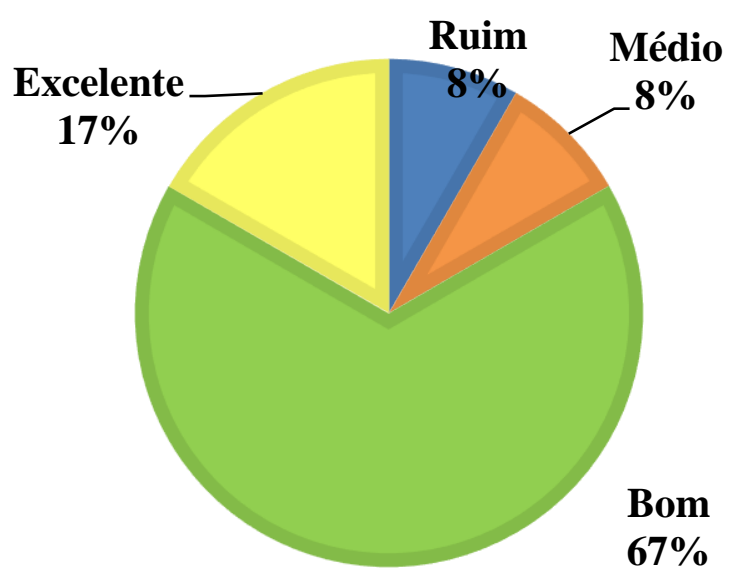

Observou-se que a maioria das fachadas $(67 \%)$ foram classificadas com estado de conservação Bom. Uma fachada foi classificada como Ruim e uma como Médio, representando apenas $8 \%$ do total. Com classificação Excelente, houveram duas fachadas, equivalente a $17 \%$. Nenhuma fachada foi classificada com estado de conservação Péssimo.

$\mathrm{Na}$ inspeção nas fachadas dos blocos, observou-se que de forma notável a existência de diversas falhas no que diz respeito a manutenção dos mesmos. A existência de tais problemas foi confirmada por meio de dos registros das imagens onde percebeu-se que o agravamento do estado de conservação das fachadas é explícito.

Apesar da possibilidade de exame visual das patologias das fachadas, o estudo conduzido com a metodologia proposta permitiu que as mesmas fossem classificadas só estados de conservação baseado em critérios técnicos previamente estabelecidos. Diante disso, mesmo considerando a subjetividade das análises, constatou-se que os resultados encontrados para as fachadas são compatíveis com as deteriorações observadas visualmente nos blocos. 


\section{CONCLUSÃO}

Em geral, todas as fachadas apresentaram, em maior ou menor grau, manifestações patológicas, sendo que fissuras e bolor foram as mais recorrentes, estando presentes em todas as edificações.

Considerando as análises apresentadas, fica evidente que o motivo de todas as edificações amostradas apresentarem estado de conservação bom, apesar de muitas delas estarem visualmente bastante deterioradas, se deve ao fato de que a maioria das patologias encontradas apenas afetam o aspecto estético das fachadas. Isso se deve também a metodologia utilizada que é qualitativa, e não quantitativa, já que leva em consideração a gravidade das patologias, e não sua quantidade e extensão.

Desse modo, fica evidente que a metodologia qualitativa utilizada não consegue refletir de forma adequada o estado atual das fachadas dos blocos da Universidade. A quantidade de manifestações patológicas é expressiva nas fachadas, devendo, portanto, ser aplicada uma metodologia que apresente um resultado mais coerente com a realidade observada in loco. Portanto, nesse caso, é apropriado que se aplique de uma metodologia quantitativa seria mais adequada.

Apesar disso, ao se comparar numericamente os resultados das edificações, a Biblioteca foi a mais deteriorada, enquanto o LEME foi a mais conservada. Essa comparação é condizente com a realidade observada em campo, em função das manifestações patológicas visíveis. Porém, ressalta-se que a análise visual se mostra pouco confiável, visto que no caso específico da Biblioteca, observou-se uma grande deterioração dos revestimentos, o que sugeriria uma classificação ruim para a fachada. Porém, apesar disso, o método aplicado indicou para a fachada um estado de conservação Médio.

O que se pode concluir é que, ao se deparar apenas com a inspeção visual, muitas edificações, podem ser classificadas como boas ou ruins de acordo com o grau de deterioração observado pelo pesquisador. Nesse caso, refletindo uma avaliação mais subjetiva. Porém, como metodologia aplicada considera uma avaliação mais criteriosa por meio da análise das incidências de anomalias e o grau de gravidade das mesmas.

Nesse sentido, a metodologia se mostra adequada para direcionar e priorizar serviços de manutenção pois pode-se concluir que o bloco da Biblioteca é aquele que necessita de intervenções de reparação com maior urgência, em seguida o CCBS. O LEME é o mais conservado, não apresentando necessidade de intervenção imediata, apenas de manutenções periódicas para mantê-lo com o atual estado de conservação.

Vale lembrar que a Biblioteca e o CCBS, desde sua construção, ambos em 1997, nunca passaram por nenhum tipo de manutenção nas fachadas. Isso se deve principalmente ao fato de a Unioeste ser uma instituição pública, onde os recursos para realização de manutenções são escassos, além da dificuldade de manter sistemas de manutenção periódicos.

O método de avaliação do estado de conservação de edifícios tem como por base o Método de Avaliação do Estado de Conservação de Imóveis, elaborado a partir da realidade portuguesa. Assim, por meio da pesquisa realizada, considera-se a necessidade de que o mesmo seja aperfeiçoado, em especial na forma de classificar a gravidade das manifestações patológicas, para conseguir refletir melhor a realidade brasileira.

\section{REFERÊNCIAS}

ALMEIDA, J. Avaliação do estado de conservação de fachadas e ensaios de arrancamento de elementos em aço de pré-esforço colados com resina epoxy a provetes de pedra natural. 2012. Relatório de estágio de Mestrado. Instituto Superior de Engenharia do Porto, Instituto Politécnico do Porto, Portugal. 
ASSOCIAÇÃO BRASILEIRA DE NORMAS TÉCNICAS. NBR 15575: Edificações habitacionais: desempenho. Rio de Janeiro, 2013.

GASPAR, P. L.; BRITO, J. Assessment of the overall degradation level of an element, based on field data. In: 10DBMC - International Conference On Durability of Building Materials and Components. Lyon, França. 17 - 20. Abril, 2005.

GUIMARÃES, L. E. Avaliação comparativa de grau de deterioração de edificações Estudo de caso: prédios pertencentes à Universidade Federal de Goiás. 2003. 265f. Dissertação de Mestrado. Escola de Engenharia Civil, Universidade Federal de Goiás, Brasil.

MAEC. Método de avaliação do estado de conservação de edifícios. Instruções de aplicação e ficha de avaliação. Ministério Obras Públicas, Transportes e Comunicações. Outubro de 2006. LNEC. Lisboa.

MENIN, T. F. S. Levantamento de manifestações patológicas em uma edificação pública: Estudo de caso na Universidade Estadual do Oeste do Paraná - Campus Cascavel. 2016. 114f. Trabalho de Conclusão de Curso (Graduação). Universidade Estadual do Oeste do Paraná, Cascavel, Brasil.

OLIVEIRA, D. F. Levantamento de causas de patologias na construção civil. 2013a. $96 \mathrm{f}$. Trabalho de Conclusão de Curso (Graduação) - Curso de Engenharia Civil, Universidade Federal do Rio de Janeiro, Rio de Janeiro.

OLIVEIRA, M. A. de. Método de avaliação de necessidades e prioridades de reabilitação de edifícios de instituições federais de ensino superior.2013b. 231f. Dissertação de Mestrado. Escola de Engenharia Civil, Universidade Federal de Goiás, Brasil.

PEDRO, J. B.; VILHENA, A.; PAIVA, J. V. de. Métodos de avaliação do estado de conservação de edifícios desenvolvidos no LNEC: Características e possibilidades de aplicação. Revista Engenharia Civil, Universidade do Minho, Lisboa, v. 42, p.5-18, jan. 2012. Disponível em <http://www.civil.uminho.pt/revista/artigos/n42/Pág.5-18.pdf>. Acesso em 01 Jul 2017.

SILVA, M. N. B. Avaliação quantitativa da degradação e vida útil de revestimentos de fachada - Aplicação ao caso de Brasília/DF. 2014. 198f. Tese (Doutorado em Estruturas e Construção Civil) - Departamento de Engenharia Civil e Ambiental, Universidade de Brasília, Brasília - DF.

VILHENA, A. Método de avaliação do estado de conservação de edifícios. Análise, diagnóstico e contributos para o seu aperfeiçoamento. Desenvolvimento de ferramentas complementares para a definição de estratégias de reabilitação. 2007. Plano de Tese de Doutoramento, LNEC, Lisboa. 